\title{
Quasilinearization for the Boundary Value Problem of Second-Order Singular Differential System
}

\author{
Peiguang Wang' and Tiantian Kong ${ }^{2}$ \\ ${ }^{1}$ College of Electronic and Information Engineering, Hebei University, Baoding 071002, China \\ ${ }^{2}$ College of Mathematics and Computer Science, Hebei University, Baoding 071002, China \\ Correspondence should be addressed to Peiguang Wang; pgwang@mail.hbu.edu.cn
}

Received 23 August 2013; Accepted 25 November 2013

Academic Editor: Yong Hong Wu

Copyright (c) 2013 P. Wang and T. Kong. This is an open access article distributed under the Creative Commons Attribution License, which permits unrestricted use, distribution, and reproduction in any medium, provided the original work is properly cited.

\begin{abstract}
We study the boundary value problems of second-order singular differential equations. At first, we reduce the BVPs to initial value problems of first-order singular integrodifferential equations and then we employ the quasilinearization method in studying the IVPs and obtain two monotone iterative sequences, which converge uniformly and quadratically to the unique solution of the IVPs. Finally, we get the similar result for the given BVPs.
\end{abstract}

\section{Introduction}

It is well known that quasilinearization method is a powerful tool for proving the existence of approximate solutions of nonlinear systems and the converge quadratically to the unique solution of the given problems (see [1]). Recently, Abd-Ellateef Kamar et al. investigated the first-order singular systems of differential equations with initial value problem [2]. In [3], Wang and Liu developed monotone iterative technique combined with the method of upper and lower solutions for studying the second-order singular systems with boundary value problems (BVPs).

In this paper, we extend quasilinearization method to study the second-order singular systems with the boundary conditions:

$$
A \ddot{X}=f(t, X, \dot{X}), \quad \dot{X}(0)=X_{0}, \quad X(b)=X_{1},
$$

where $A \in R^{n \times n}$ is a singular matrix, $f \in C\left[I \times R^{n} \times R^{n}, R^{n}\right]$, $I=[0, b]$, and $X_{0}$ and $X_{1}$ are two constant vectors. By using the existence result [4] for linear singular systems and the comparison result [5],we investigate two monotone iterative sequences which converge uniformly and quadratically to the solution of the problem.

\section{Preliminaries}

Consider the following initial value problem:

$$
-A \dot{U}=f(t, S U,-U), \quad U(0)=-X_{0},
$$

where $A$ is a singular $n \times n$ matrix, $f \in C\left[I \times R^{n} \times R^{n}, R^{n}\right]$, $I=[0, b], S U=X_{1}+\int_{t}^{b} U(t) d s$ is an increasing operator, and $X_{0}$ is a constant vector.

In order to obtain two monotone sequences, we introduce an existence result for the corresponding linear singular systems and a comparison result.

The existence of the solution of the linear initial value problem of the form

$$
-A \dot{U}+M(t) U=g(t), \quad U(0)=-X_{0}
$$

is well known and is given by the following lemma. 
Lemma 1 (see [4]). Assume that the nonhomogeneous linear system (3) exists and if

(i) there exists a $\lambda \in R$ such that $(-\lambda A+M(t))^{-1}$ exists,

(ii) $X_{0}$ is the solution of $\left(I-\widehat{A} \widehat{A}^{D}\right)\left(-X_{0}-\widehat{W}(0)\right)=0$, where

$$
\begin{gathered}
\widehat{A}=(-A+M(t))^{-1}(-A), \\
\widehat{M}=(-A+M(t))^{-1} M(t), \\
\widehat{g}(t)=(-A+M(t))^{-1} g(t), \\
\widehat{W}(t)=\left(I-\widehat{A}^{D} \widehat{A}\right) \widehat{M}^{D} \widehat{g}(t),
\end{gathered}
$$

and $\widehat{A}^{D}, \widehat{M}^{D}$ are the Drazin inverses of $\widehat{A}, \widehat{M}(t)$, respectively, then the solution is given by

$$
\begin{aligned}
U(t)= & e^{-\widehat{A}^{D} \widehat{M} t} \widehat{A}^{D} \widehat{A}\left(-X_{0}\right)+e^{-\widehat{A}^{D} \widehat{M} t} \int_{0}^{t} e^{\widehat{A}^{D} \widehat{M} s} \widehat{A}^{D} \widehat{g}(s) d s \\
& +\left(I-\widehat{A}^{D} \widehat{A}\right) \widehat{M}^{D} \widehat{g}(t) .
\end{aligned}
$$

Now one gives the following assumptions for convenience.

$\left(H_{2.1}\right)$ Let $A$ and $M(t)$ be matrices such that $(-\lambda A+M(t))^{-1}$ exists and is nonnegative for some $\lambda \in R^{1}$. Suppose further that $T, T^{-1}$ exist and are nonnegative, such that

$$
T^{-1} \widehat{A} T=\left(\begin{array}{cc}
C & 0 \\
0 & 0
\end{array}\right), \quad T^{-1} \widehat{M} T=\left(\begin{array}{cc}
I_{1}-\lambda C & 0 \\
0 & I_{2}
\end{array}\right)
$$

where $\widehat{A}=(-\lambda A+M(t))^{-1} A, \widehat{M}=(-\lambda A+$ $M(t))^{-1} M(t)$, and $C$ is a diagonal square matrix with $C^{-1}>0$ and $C^{-1}\left(I_{1}-\lambda C\right)>0$.

$\left(H_{2.2}\right)$ There exist $\alpha_{0}(t), \beta_{0}(t) \in C^{1}\left[I, R^{n}\right]$ with $\alpha_{0}(t) \leq \beta_{0}(t)$ on $I$, such that

$$
\begin{array}{ll}
-A \dot{\alpha}_{0} \leq f\left(t, S \alpha_{0},-\alpha_{0}\right), & \alpha_{0}(0) \leq-X_{0}, \\
-A \dot{\beta}_{0} \geq f\left(t, S \beta_{0},-\beta_{0}\right), & \beta_{0}(0) \geq-X_{0} .
\end{array}
$$

$\left(H_{2.3}\right)$ All second-order derivatives of $f(t, X, U)$ exist and are bounded, $f(t, X, U)$ is convex in $X$ for each $(t, U)$, $f(t, X, U)$ is convex in $U$ for each $(t, X), f_{x}(t, X, U)$ is nonincreasing in $U$ for each $(t, X)$, and $f_{y}(t, X, U)$ is nonincreasing in $X$ for each $(t, U)$.

$\left(H_{2.4}\right)$ Moreover $M(t)=-\left[f_{x}(t, S \eta,-\eta) b-f_{y}(t, S \eta,-\eta)\right]$.

To obtain the results, one needs the following comparison theorem.

Lemma 2. Let $-A \dot{P}+M(t) P \leq 0$ such that $A$ and $M(t)$ satisfy assumptions $\left(H_{2.1}\right)$ and $\left(H_{2.4}\right)$. Then $P(0) \leq 0$ implies $P(t) \leq 0$ on $I$.

The proof is similar to [5] and one omits it.

\section{Main Results}

Firstly, we develop the following result which is important for the final result.

Theorem 3. Suppose that assumptions $\left(H_{2.1}\right)-\left(H_{2.4}\right)$ hold and, for $X_{0} \in R^{n}$,

(i) $\left(I-\widehat{A} \widehat{A}^{D}\right)\left(-X_{0}-\widehat{W}(0)\right)=0$, where $\widehat{W}(t)=(I-$ $\left.\widehat{A}^{D} \widehat{A}\right) \widehat{M}^{D} \widehat{g}(t)$.

Then there exist two monotone sequences $\left\{\alpha_{n}\right\}$ and $\left\{\beta_{n}\right\}$, which converge uniformly on I to the unique solution of problem (2) and the convergence rate is quadratic.

Proof. From $\left(H_{2.3}\right)$ we find that $f_{x x}(t, X, U) \geq 0$, and

$$
f\left(t, X_{1}, U\right) \geq f\left(t, X_{2}, U\right)+f_{x}\left(t, X_{2}, U\right)\left(X_{1}-X_{2}\right),
$$

for all $X_{1}, X_{2}, U \in R^{n}$ and $t \in I$. The convexity of $f(t, X, U)$ in $U$ implies that

$$
f\left(t, X, U_{1}\right) \geq f\left(t, X, U_{2}\right)+f_{y}\left(t, X, U_{2}\right)\left(U_{1}-U_{2}\right),
$$

for $U_{1}, U_{2} \in R^{n}, t \in I$, and $X \in R^{n}$.

Now consider the following linear problems. For $k=$ $0,1,2,3 \ldots$,

$$
\begin{aligned}
-A \dot{\alpha}_{k+1}= & f\left(t, S \alpha_{k},-\alpha_{k}\right)+f_{x}\left(t, S \alpha_{k},-\alpha_{k}\right)\left(S \alpha_{k+1}-S \alpha_{k}\right) \\
& +f_{y}\left(t, S \alpha_{k},-\alpha_{k}\right)\left(-\alpha_{k+1}+\alpha_{k}\right), \quad \alpha_{k+1}(0)=-X_{0},
\end{aligned}
$$

$$
\begin{aligned}
-A \dot{\beta}_{k+1}= & f\left(t, S \beta_{k},-\beta_{k}\right)+f_{x}\left(t, S \alpha_{k},-\alpha_{k}\right)\left(S \beta_{k+1}-S \beta_{k}\right) \\
& +f_{y}\left(t, S \alpha_{k},-\alpha_{k}\right)\left(-\beta_{k+1}+\beta_{k}\right), \quad \beta_{k+1}(0)=-X_{0} .
\end{aligned}
$$

For (10), set

$$
\begin{aligned}
g(t, S x,-x) \equiv & f\left(t, S \alpha_{0},-\alpha_{0}\right)+f_{x}\left(t, S \alpha_{0},-\alpha_{0}\right)\left(S x-S \alpha_{0}\right) \\
& +f_{y}\left(t, S \alpha_{0},-\alpha_{0}\right)\left(-x+\alpha_{0}\right) .
\end{aligned}
$$

Then, we obtain that $-A \dot{\alpha}_{0} \leq f\left(t, S \alpha_{0},-\alpha_{0}\right)=g\left(t, S \alpha_{0},-\alpha_{0}\right)$. Furthermore, we can get

$$
\begin{aligned}
-A \dot{\beta}_{0} \geq & f\left(t, S \beta_{0},-\beta_{0}\right) \\
\geq & f\left(t, S \alpha_{0},-\alpha_{0}\right)+f_{x}\left(t, S \alpha_{0},-\alpha_{0}\right)\left(S \beta_{0}-S \alpha_{0}\right) \\
& +f_{y}\left(t, S \alpha_{0},-\alpha_{0}\right)\left(-\beta_{0}+\alpha_{0}\right) \\
= & g\left(t, S \beta_{0},-\beta_{0}\right) .
\end{aligned}
$$

Hence $\alpha_{0}$ and $\beta_{0}$ are lower and upper solutions of (12), respectively. Thus (12) has a solution $\alpha_{1}$ on $I$, and we have $\alpha_{0} \leq \alpha_{1} \leq \beta_{0}$. Similarly, set

$$
\begin{aligned}
g^{*}(t, S x,-x) \equiv & f\left(t, S \beta_{0},-\beta_{0}\right)+f_{x}\left(t, S \alpha_{0},-\alpha_{0}\right)\left(S x-S \beta_{0}\right) \\
& +f_{y}\left(t, S \alpha_{0},-\alpha_{0}\right)\left(-x+\beta_{0}\right) .
\end{aligned}
$$


Then we obtain that (14) has a solution $\beta_{1}$ on $I$, and we have $\alpha_{0} \leq \beta_{1} \leq \beta_{0}$.

Now we claim that

$$
\alpha_{0} \leq \alpha_{1} \leq \beta_{1} \leq \beta_{0} \text { on } I .
$$

Let $p=\alpha_{1}-\beta_{1}$. We get that

$$
\begin{aligned}
& -A \dot{p}=-A \dot{\alpha}_{1}-\left(-A \dot{\beta}_{1}\right) \\
& =f\left(t, S \alpha_{0},-\alpha_{0}\right)+f_{x}\left(t, S \alpha_{0},-\alpha_{0}\right)\left(S \alpha_{1}-S \alpha_{0}\right) \\
& +f_{y}\left(t, S \alpha_{0},-\alpha_{0}\right)\left(-\alpha_{1}+\alpha_{0}\right) \\
& -f\left(t, S \beta_{0},-\beta_{0}\right)-f_{x}\left(t, S \alpha_{0},-\alpha_{0}\right)\left(S \beta_{1}-S \beta_{0}\right) \\
& -f_{y}\left(t, S \alpha_{0},-\alpha_{0}\right)\left(-\beta_{1}+\beta_{0}\right) \\
& =f\left(t, S \alpha_{0},-\alpha_{0}\right)-f\left(t, S \beta_{0},-\alpha_{0}\right) \\
& +f\left(t, S \beta_{0},-\alpha_{0}\right)-f\left(t, S \beta_{0},-\beta_{0}\right) \\
& +f_{x}\left(t, S \alpha_{0},-\alpha_{0}\right)\left(S \alpha_{1}-S \alpha_{0}\right) \\
& +f_{y}\left(t, S \alpha_{0},-\alpha_{0}\right)\left(-\alpha_{1}+\alpha_{0}\right) \\
& -f_{x}\left(t, S \alpha_{0},-\alpha_{0}\right)\left(S \beta_{1}-S \beta_{0}\right) \\
& -f_{y}\left(t, S \alpha_{0},-\alpha_{0}\right)\left(-\beta_{1}+\beta_{0}\right) \\
& \leq f_{x}\left(t, S \alpha_{0},-\alpha_{0}\right)\left(S \alpha_{0}-S \beta_{0}\right) \\
& +f_{y}\left(t, S \alpha_{0},-\alpha_{0}\right)\left(-\alpha_{0}+\beta_{0}\right) \\
& +f_{x}\left(t, S \alpha_{0},-\alpha_{0}\right)\left(S \alpha_{1}-S \alpha_{0}\right) \\
& +f_{y}\left(t, S \alpha_{0},-\alpha_{0}\right)\left(-\alpha_{1}+\alpha_{0}\right) \\
& -f_{x}\left(t, S \beta_{0},-\beta_{0}\right)\left(S \beta_{1}-S \beta_{0}\right) \\
& -f_{y}\left(t, S \beta_{0},-\beta_{0}\right)\left(-\beta_{1}+\beta_{0}\right) \\
& \leq f_{x}\left(t, S \alpha_{0},-\alpha_{0}\right) \\
& \times\left(S \alpha_{0}-S \beta_{0}+S \alpha_{1}-S \alpha_{0}-S \beta_{1}+S \beta_{0}\right) \\
& +f_{y}\left(t, S \alpha_{0},-\alpha_{0}\right) \\
& \times\left(-\alpha_{0}+\beta_{0}-\alpha_{1}+\alpha_{0}+\beta_{1}-\beta_{0}\right) \\
& =f_{x}\left(t, S \alpha_{0},-\alpha_{0}\right)\left(S \alpha_{1}-S \beta_{1}\right) \\
& -f_{y}\left(t, S \alpha_{0},-\alpha_{0}\right)\left(\alpha_{1}-\beta_{1}\right) \leq-M(t)\left(\alpha_{1}-\beta_{1}\right) \text {. }
\end{aligned}
$$

Noticing that $p(0)=\alpha_{1}(0)-\beta_{1}(0) \leq 0$, we get $\alpha_{1}(t) \leq \beta_{1}(t)$, on $I$.

Now, assume that, for $n=0,1,2, \ldots, k,(10)$ and (11) admit solutions $\alpha_{n}$ and $\beta_{n}$, respectively, such that

$$
\alpha_{0} \leq \alpha_{1} \leq \cdots \leq \alpha_{k} \leq \beta_{k} \leq \cdots \leq \beta_{1} \leq \beta_{0}, \quad \text { on } I .
$$

Then setting $n=k$ in (10) and (11), we observe that the assumptions in Theorem 3 are satisfied. Thus, there exist solutions $\alpha_{k+1}(t)$ and $\beta_{k+1}(t)$ for (10) and (11), respectively, and we now will show that the following relation

$$
\alpha_{k}(t) \leq \alpha_{k+1}(t) \leq \beta_{k+1}(t) \leq \beta_{k}(t), \quad t \in I,
$$

holds. Firstly, we can easily know that $\alpha_{k}(t) \leq \alpha_{k+1}(t) \leq \beta_{k}(t)$ and $\alpha_{k}(t) \leq \beta_{k+1}(t) \leq \beta_{k}(t)$.

To prove that $\alpha_{k+1} \leq \beta_{k+1}$, consider $p=\alpha_{k+1}-\beta_{k+1}$. Then

$$
\begin{aligned}
& -A \dot{p}=-A \dot{\alpha}_{k+1}-\left(-A \dot{\beta}_{k+1}\right) \\
& =f\left(t, S \alpha_{k},-\alpha_{k}\right)+f_{x}\left(t, S \alpha_{k},-\alpha_{k}\right)\left(S \alpha_{k+1}-S \alpha_{k}\right) \\
& +f_{y}\left(t, S \alpha_{k},-\alpha_{k}\right)\left(-\alpha_{k+1}+\alpha_{k}\right) \\
& -f\left(t, S \beta_{k},-\beta_{k}\right)-f_{x}\left(t, S \beta_{k},-\beta_{k}\right)\left(S \beta_{k+1}-S \beta_{k}\right) \\
& -f_{y}\left(t, S \beta_{k},-\beta_{k}\right)\left(-\beta_{k+1}+\beta_{k}\right) \\
& =f\left(t, S \alpha_{k},-\alpha_{k}\right)-f\left(t, S \beta_{k},-\alpha_{k}\right) \\
& +f\left(t, S \beta_{k},-\alpha_{k}\right)-f\left(t, S \beta_{k},-\beta_{k}\right) \\
& +f_{x}\left(t, S \alpha_{k},-\alpha_{k}\right)\left(S \alpha_{k+1}-S \alpha_{k}\right) \\
& +f_{y}\left(t, S \alpha_{k},-\alpha_{k}\right)\left(-\alpha_{k+1}+\alpha_{k}\right) \\
& -f_{x}\left(t, S \beta_{k},-\beta_{k}\right)\left(S \beta_{k+1}-S \beta_{k}\right) \\
& -f_{y}\left(t, S \beta_{k},-\beta_{k}\right)\left(-\beta_{k+1}+\beta_{k}\right) \\
& \leq f_{x}\left(t, S \alpha_{k},-\alpha_{k}\right)\left(S \alpha_{k}-S \beta_{k}\right) \\
& +f_{y}\left(t, S \beta_{k},-\alpha_{k}\right)\left(-\alpha_{k}+\beta_{k}\right) \\
& +f_{x}\left(t, S \alpha_{k},-\alpha_{k}\right)\left(S \alpha_{k+1}-S \alpha_{k}\right) \\
& +f_{y}\left(t, S \alpha_{k},-\alpha_{k}\right)\left(-\alpha_{k+1}+\alpha_{k}\right) \\
& -f_{x}\left(t, S \beta_{k},-\beta_{k}\right)\left(S \beta_{k+1}-S \beta_{k}\right) \\
& -f_{y}\left(t, S \beta_{k},-\beta_{k}\right)\left(-\beta_{k+1}+\beta_{k}\right) \\
& \leq f_{x}\left(t, S \alpha_{k},-\alpha_{k}\right) \\
& \times\left(S \alpha_{k}-S \beta_{k}+S \alpha_{k+1}-S \alpha_{k}-S \beta_{k+1}+S \beta_{k}\right) \\
& +f_{y}\left(t, S \alpha_{k},-\alpha_{k}\right) \\
& \times\left(-\alpha_{k}+\beta_{k}-\alpha_{k+1}+\alpha_{k}-\beta_{k+1}+\beta_{k}\right) \\
& =f_{x}\left(t, S \alpha_{k},-\alpha_{k}\right)\left(S \alpha_{k+1}-S \beta_{k+1}\right) \\
& +f_{y}\left(t, S \alpha_{k},-\alpha_{k}\right)\left(-\alpha_{k+1}+\beta_{k+1}\right)=-M(t) p .
\end{aligned}
$$

We know that $p(0)=\alpha_{k+1}(0)-\beta_{k+1}(0) \leq 0$. Hence, from Lemma 2, we deduce that $\alpha_{k+1}(t) \leq \beta_{k+1}(t)$, on $I$. Thus, we have monotone sequences $\left\{\alpha_{n}\right\},\left\{\beta_{n}\right\}$ such that

$$
\alpha_{0} \leq \alpha_{1} \leq \cdots \leq \alpha_{n} \leq \beta_{n} \leq \cdots \leq \beta_{1} \leq \beta_{0}, \quad \text { on } I .
$$

Now, employing Ascoli-Arzela's theorem we conclude that the sequences converge uniformly and monotonically to the unique solution $U(t)$ of (2) on $I$. 
To show that the convergence rate is quadratic, we begin with $p_{n+1}=x-\alpha_{n+1}$. Then

$$
\begin{aligned}
& -A \dot{p}_{n+1}=-A \dot{x}-\left(-A \dot{\alpha}_{n+1}\right) \\
& =f(t, S x,-x)-f\left(t, S \alpha_{n},-\alpha_{n}\right) \\
& -f_{x}\left(t, S \alpha_{n},-\alpha_{n}\right)\left(S \alpha_{n+1}-S \alpha_{n}\right) \\
& -f_{y}\left(t, S \alpha_{n},-\alpha_{n}\right)\left(-\alpha_{n+1}+\alpha_{n}\right) \\
& =f(t, S x,-x)-f\left(t, S \alpha_{n},-\alpha_{n}\right) \\
& -f_{x}\left(t, S \alpha_{n},-\alpha_{n}\right)\left(S \alpha_{n+1}-S x+S x-S \alpha_{n}\right) \\
& -f_{y}\left(t, S \alpha_{n},-\alpha_{n}\right)\left(-\alpha_{n+1}+x-x+\alpha_{n}\right) \\
& =f(t, S x,-x)-f\left(t, S \alpha_{n},-\alpha_{n}\right) \\
& +f_{x}\left(t, S \alpha_{n},-\alpha_{n}\right) S p_{n+1}-f_{x}\left(t, S \alpha_{n},-\alpha_{n}\right) S p_{n} \\
& -f_{y}\left(t, S \alpha_{n},-\alpha_{n}\right) p_{n+1}+f_{y}\left(t, S \alpha_{n},-\alpha_{n}\right) p_{n} \\
& =f_{x}\left(t, S \alpha_{n},-\alpha_{n}\right) S p_{n+1}-f_{y}\left(t, S \alpha_{n},-\alpha_{n}\right) p_{n+1} \\
& +f(t, S x,-x)-f\left(t, S \alpha_{n},-\alpha_{n}\right) \\
& -f_{x}\left(t, S \alpha_{n},-\alpha_{n}\right) S p_{n}+f_{y}\left(t, S \alpha_{n},-\alpha_{n}\right) p_{n} \\
& \leq-M(t) p+f(t, S x,-x) \\
& -f\left(t, S \alpha_{n},-\alpha_{n}\right)-f_{x}\left(t, S \alpha_{n},-\alpha_{n}\right) S p_{n} \\
& +f_{y}\left(t, S \alpha_{n},-\alpha_{n}\right) p_{n} \\
& =-M(t) p+f(t, S x,-x)-f\left(t, S \alpha_{n},-x\right) \\
& +f\left(t, S \alpha_{n},-x\right)-f\left(t, S \alpha_{n},-\alpha_{n}\right) \\
& -f_{x}\left(t, S \alpha_{n},-\alpha_{n}\right) S p_{n}+f_{y}\left(t, S \alpha_{n},-\alpha_{n}\right) p_{n} \\
& =-M(t) p \\
& +\int_{0}^{1} f_{x}\left(t, \sigma S x+(1-\sigma) S \alpha_{n},-x\right)\left(S x-S \alpha_{n}\right) d \sigma \\
& +\int_{0}^{1} f_{y}\left(t, S \alpha_{n}, \sigma(-x)+(1-\sigma)\left(-\alpha_{n}\right)\right) \\
& \times\left(-x+\alpha_{n}\right) d \sigma \\
& -f_{x}\left(t, S \alpha_{n},-\alpha_{n}\right) S p_{n}+f_{y}\left(t, S \alpha_{n},-\alpha_{n}\right) p_{n} \\
& =-M(t) p+\int_{0}^{1}\left[f_{x}\left(t, \sigma S x+(1-\sigma) S \alpha_{n},-x\right)\right. \\
& \left.-f_{x}\left(t, S \alpha_{n},-\alpha_{n}\right)\right] S p_{n} d \sigma \\
& -\int_{0}^{1}\left[f_{y}\left(t, S \alpha_{n}, \sigma(-x)+(1-\sigma)\left(-\alpha_{n}\right)\right)\right. \\
& \left.-f_{y}\left(t, S \alpha_{n},-\alpha_{n}\right)\right] p_{n} d \sigma \\
& =-M(t) p+A_{1}+B_{1} \text {, }
\end{aligned}
$$

where

$$
\begin{gathered}
A_{1}=\int_{0}^{1}\left[f_{x}\left(t, \sigma S x+(1-\sigma) S \alpha_{n},-x\right)\right. \\
\left.-f_{x}\left(t, S \alpha_{n},-\alpha_{n}\right)\right] S p_{n} d \sigma \\
B_{1}=-\int_{0}^{1}\left[f_{y}\left(t, S \alpha_{n}, \sigma(-x)+(1-\sigma)\left(-\alpha_{n}\right)\right)\right. \\
\left.-f_{y}\left(t, S \alpha_{n},-\alpha_{n}\right)\right] p_{n} d \sigma .
\end{gathered}
$$

Set $\xi_{1}=\sigma S x+(1-\sigma) S \alpha_{n}$. Then

$$
\begin{aligned}
A_{1}= & \int_{0}^{1}\left[f_{x}\left(t, \xi_{1},-x\right)-f_{x}\left(t, S \alpha_{n},-\alpha_{n}\right)\right] S p_{n} d \sigma \\
= & \int_{0}^{1}\left[f_{x}\left(t, \xi_{1},-x\right)-f_{x}\left(t, S \alpha_{n},-x\right)+f_{x}\left(t, S \alpha_{n},-x\right)\right. \\
& \left.\quad-f_{x}\left(t, S \alpha_{n},-\alpha_{n}\right)\right] S p_{n} d \sigma \\
=\iint_{0}^{1}\left[f_{x x}\left(t, \tau \xi_{1}+(1-\tau) S \alpha_{n},-x\right)\left(\xi_{1}-S \alpha_{n}\right)\right. & \quad+f_{x y}\left(t, S \alpha_{n}, \tau(-x)+(1-\tau)\left(-\alpha_{n}\right)\right) \\
\quad & \left.\quad\left(-x+\alpha_{n}\right)\right] S p_{n} d \tau d \sigma \\
=\iint_{0}^{1}\left[f_{x x}\left(t, \xi_{2},-x\right) \sigma\left(S x-S \alpha_{n}\right)\right. & \left.\quad-f_{x y}\left(t, S \alpha_{n}, \xi_{3}\right)\left(x-\alpha_{n}\right)\right] S p_{n} d \tau d \sigma \\
= & \frac{1}{2} N_{1} S p_{n}^{2}+N_{2} p_{n} S p_{n} \leq\left(\frac{1}{2} N_{1} b^{2}+N_{2} b\right) p_{n}^{2},
\end{aligned}
$$

where $\xi_{2}=\tau \xi_{1}+(1-\tau) S \alpha_{n}, \xi_{3}=\tau(-x)+(1-\tau)\left(-\alpha_{n}\right)$, $f_{x x}(t, U, X) \leq N_{1}$, and $-f_{x y}(t, U, X) \leq N_{2}$.

Next, set $\eta_{1}=\sigma(-x)+(1-\sigma)\left(-\alpha_{n}\right)$. Then

$$
\begin{gathered}
B_{1}=-\int_{0}^{1}\left[f_{y}\left(t, S \alpha_{n}, \eta_{1}\right)-f_{y}\left(t, S \alpha_{n},-\alpha_{n}\right)\right] p_{n} d \sigma \\
=-\iint_{0}^{1}\left[f_{y y}\left(t, S \alpha_{n}, \tau \eta_{1}+(1-\tau)\left(-\alpha_{n}\right)\right)\right. \\
\left.\quad \times\left(\eta_{1}+\alpha_{n}\right)\right] p_{n} d \sigma d \tau \\
=\iint_{0}^{1} f_{y y}\left(t, S \alpha_{n}, \eta_{2}\right) \sigma p_{n}^{2} d \sigma d \tau \leq \frac{1}{2} N_{3} p_{n}^{2},
\end{gathered}
$$

where $f_{y y}(t, U, X) \leq N_{3}$ and $\eta_{2}=\tau \eta_{1}+(1-\tau)\left(-\alpha_{n}\right)$.

Furthermore, we have that

$$
-A \dot{p}_{n+1} \leq-M(t) p_{n+1}+N_{4} p_{n}^{2}
$$

where $N_{4}=(1 / 2) N_{1} b^{2}+N_{2} b+(1 / 2) N_{3}$.

Using Lemma 2 , we show that $p_{n+1}(t) \leq U(t)$ on $I$, where $U(t)$ is the solution of

$$
-A \dot{U}+M(t) U=N_{4} p_{n}^{2}, \quad U(0)=0 .
$$


Thus, using Lemma 1, the solution of the previously mentioned equation is given as

$$
\begin{aligned}
U(t)= & e^{-\widehat{A}^{D} \widehat{M} t} \int_{0}^{t} e^{\widehat{A}^{D} \widehat{M} s} \widehat{A}^{D}(-\lambda A+M(s))^{-1} N_{4} p_{n}^{2}(s) d s \\
& +\left(I-\widehat{A}^{D} \widehat{A}\right) \widehat{M}^{D}(-\lambda A+M(t))^{-1} N_{4} p_{n}^{2}(t) .
\end{aligned}
$$

After taking suitable estimates, we obtain

$$
p_{n+1} \leq K_{1} p_{n}^{2}
$$

where $K_{1}=e^{-\widehat{A}^{D} \widehat{M} t} \int_{0}^{t} e^{\widehat{A}^{D} \widehat{M} s} \widehat{A}^{D}(-\lambda A+M(s))^{-1} N_{4} d s+(I-$ $\left.\widehat{A}^{D} \widehat{A}\right) \widehat{M}^{D}(-\lambda A+M(t))^{-1} N_{4}$.

Set $q_{n+1}=\beta_{n+1}-x$. Then we can get

$$
\begin{aligned}
& -A \dot{q}_{n+1}=-A \dot{\beta}_{n+1}-(-A \dot{x}) \\
& =f\left(t, S \beta_{n},-\beta_{n}\right)+f_{x}\left(t, S \alpha_{n},-\alpha_{n}\right)\left(S \beta_{n+1}-S \beta_{n}\right) \\
& +f_{y}\left(t, S \alpha_{n},-\alpha_{n}\right)\left(-\beta_{n+1}+\beta_{n}\right)-f(t, S x,-x) \\
& =f_{x}\left(t, S \alpha_{n},-\alpha_{n}\right)\left(S \beta_{n+1}-S x+S x-S \beta_{n}\right) \\
& +f_{y}\left(t, S \alpha_{n},-\alpha_{n}\right)\left(-\beta_{n+1}+x-x+\beta_{n}\right) \\
& +f\left(t, S \beta_{n},-\beta_{n}\right)-f(t, S x,-x) \\
& =f_{x}\left(t, S \alpha_{n},-\alpha_{n}\right)\left(S \beta_{n+1}-S x\right) \\
& -f_{y}\left(t, S \alpha_{n},-\alpha_{n}\right)\left(\beta_{n+1}-x\right)+f\left(t, S \beta_{n},-\beta_{n}\right) \\
& -f(t, S x,-x)-f_{x}\left(t, S \alpha_{n},-\alpha_{n}\right)\left(S \beta_{n}-S x\right) \\
& +f_{y}\left(t, S \alpha_{n},-\alpha_{n}\right)\left(\beta_{n}-x\right) \\
& \leq-M(t) q_{n+1}+f\left(t, S \beta_{n},-\beta_{n}\right)-f\left(t, S x,-\beta_{n}\right) \\
& +f\left(t, S x,-\beta_{n}\right)-f(t, S x,-x) \\
& -f_{x}\left(t, S \alpha_{n},-\alpha_{n}\right)\left(S \beta_{n}-S x\right) \\
& +f_{y}\left(t, S \alpha_{n},-\alpha_{n}\right)\left(\beta_{n}-x\right) \\
& =-M(t) q_{n+1} \\
& +\int_{0}^{1} f_{x}\left(t, \sigma S \beta_{n}+(1-\sigma) S x,-\beta_{n}\right)\left(S \beta_{n}-S x\right) d \sigma
\end{aligned}
$$

$$
\begin{aligned}
& +\int_{0}^{1} f_{y}\left(t, S x, \sigma\left(-\beta_{n}\right)+(1-\sigma)(-x)\right) \\
& \quad \times\left(-\beta_{n}+x\right) d \sigma \\
& -f_{x}\left(t, S \alpha_{n},-\alpha_{n}\right)\left(S \beta_{n}-S x\right) \\
& +f_{y}\left(t, S \alpha_{n},-\alpha_{n}\right)\left(\beta_{n}-x\right) \\
& =-M(t) q_{n+1}+\int_{0}^{1}\left[f_{x}\left(t, \sigma S \beta_{n}+(1-\sigma) S x,-\beta_{n}\right)\right. \\
& \quad-\int_{0}^{1}\left[f_{y}\left(t, S x, \sigma\left(-\beta_{n}\right)+\left(1-\sigma \alpha_{n},-\alpha_{n}\right)\right] S q_{n} d \sigma\right. \\
& \left.\quad-f_{y}\left(t, S \alpha_{n},-\alpha_{n}\right)\right] q_{n} d \sigma \\
& =-M(t) q_{n+1}+A_{2}+B_{2},
\end{aligned}
$$

in which

$$
\begin{gathered}
A_{2}=\int_{0}^{1}\left[f_{x}\left(t, \sigma S \beta_{n}+(1-\sigma) S x,-\beta_{n}\right)\right. \\
\left.-f_{x}\left(t, S \alpha_{n},-\alpha_{n}\right)\right] S q_{n} d \sigma \\
B_{2}=-\int_{0}^{1}\left[f_{y}\left(t, S x, \sigma\left(-\beta_{n}\right)+(1-\sigma)(-x)\right)\right. \\
\left.-f_{y}\left(t, S \alpha_{n},-\alpha_{n}\right)\right] q_{n} d \sigma .
\end{gathered}
$$

Set $\theta_{1}=\sigma S \beta_{n}+(1-\sigma) S x$. Then we get that

$$
\begin{aligned}
A_{2}= & \int_{0}^{1}\left[f_{x}\left(t, \theta_{1},-\beta_{n}\right)-f_{x}\left(t, S \alpha_{n},-\alpha_{n}\right)\right] S q_{n} d \sigma \\
= & \int_{0}^{1}\left[f_{x}\left(t, \theta_{1},-\beta_{n}\right)-f_{x}\left(t, S \alpha_{n},-\beta_{n}\right)\right. \\
& \left.\quad+f_{x}\left(t, S \alpha_{n},-\beta\right)-f_{x}\left(t, S \alpha_{n},-\alpha_{n}\right)\right] S q_{n} d \sigma \\
= & \iint_{0}^{1}\left[f_{x x}\left(t, \tau \theta_{1}+(1-\tau) S \alpha_{n},-\beta_{n}\right)\left(\theta_{1}-S \alpha_{n}\right)\right. \\
& \quad+f_{x y}\left(t, S \alpha_{n}, \tau\left(-\beta_{n}\right)+(1-\tau)\left(-\alpha_{n}\right)\right) \\
= & \iint_{0}^{1}\left[f_{x x}\left(t, \theta_{2},-\beta_{n}\right)\left(\sigma q_{n}+p_{n}\right)\right] S q_{n} d \sigma d \tau
\end{aligned}
$$

where $\theta_{2}=\tau \theta_{1}+(1-\tau) S \alpha_{n}$ and $\theta_{3}=\tau\left(-\beta_{n}\right)+(1-\tau)\left(-\alpha_{n}\right)$. 
Similarly, set $\vartheta_{1}=\sigma\left(-\beta_{n}\right)+(1-\sigma)(-x)$. Then

$$
\begin{aligned}
& B_{2}=-\int_{0}^{1}\left[f_{y}\left(t, S x, \vartheta_{1}\right)-f_{y}\left(t, S \alpha_{n},-\alpha_{n}\right)\right] q_{n} d \sigma \\
&=-\int_{0}^{1}\left[f_{y}\left(t, S x, \vartheta_{1}\right)-f_{y}\left(t, S \alpha_{n}, \vartheta_{1}\right)\right.\left.\quad+f_{y}\left(t, S \alpha_{n}, \vartheta_{1}\right)-f_{y}\left(t, S \alpha_{n},-\alpha_{n}\right)\right] q_{n} d \sigma \\
&=-\iint_{0}^{1}\left[f_{y x}\left(t, \tau S x+(1-\tau) S \alpha_{n}, \vartheta_{1}\right)\left(S x-S \alpha_{n}\right)\right. \\
& \quad+f_{y y}\left(t, S \alpha_{n}, \tau \vartheta_{1}+(1-\tau)\left(-\alpha_{n}\right)\right) \\
&\left.\quad \times\left(\vartheta_{1}+\alpha_{n}\right)\right] q_{n} d \sigma d \tau \\
&=-\iint_{0}^{1}\left[f_{y x}\left(t, \vartheta_{2}, \vartheta_{1}\right) S p_{n}+f_{y y}\left(t, S \alpha_{n}, \vartheta_{3}\right)\right. \\
&\left.\quad \times\left(\sigma\left(-\beta_{n}+x\right)-\left(x-\alpha_{n}\right)\right)\right] q_{n} d \sigma d \tau \\
& \leq \iint_{0}^{1}\left[-f_{y x}\left(t, \vartheta_{2}, \vartheta_{1}\right) S p_{n}\right. \\
&\left.+f_{y y}\left(t, S \alpha_{n}, \vartheta_{3}\right)\left(\sigma q_{n}+p_{n}\right)\right] q_{n} d \sigma d \tau \\
& 1 \\
& \leq N_{5} b q_{n} p_{n}+\frac{1}{2} N_{3} q_{n}^{2}+N_{3} p_{n} q_{n} \\
&\left.+N_{3}\right) q_{n}^{2}+\frac{1}{2}\left(b N_{5}+N_{3}\right) p_{n}^{2},
\end{aligned}
$$

where $\vartheta_{2}=\tau S x+(1-\tau) S \alpha_{n}, \vartheta_{3}=\tau \vartheta_{1}+(1-\tau)\left(-\alpha_{n}\right)$, and $-f_{y x}(t, X, U) \leq N_{5}$.

Then we get that

$$
-A \dot{q}_{n+1} \leq-M(t) q_{n+1}+N_{6} q_{n}^{2}+N_{7} p_{n}^{2}
$$

where $N_{6}=\left(N_{1}+(3 / 2) N_{2}\right) b+\left((1 / 2) b N_{5}+N_{3}\right)$ and $N_{7}=$ $(1 / 2) b\left(N_{1}+N_{2}\right)+(1 / 2)\left(b N_{5}+N_{3}\right)$. Thus we have that

$$
\begin{aligned}
q_{n+1}(t) & \\
\leq U(t)= & e^{-\widehat{A}^{D} \widehat{M} t} \int_{0}^{t} e^{\widehat{A}^{D} \widehat{M} s} \widehat{A}^{D}(-\lambda A+M(s))^{-1} \\
& \times\left(N_{6} q_{n}(s)^{2}+N_{7} p_{n}(s)^{2}\right) d s \\
& +\left(I-\widehat{A}^{D} \widehat{A}\right) \widehat{M}^{D}(-\lambda A+M(t))^{-1} N_{6} q_{n}(t)^{2} \\
& +N_{7} p_{n}(t)^{2} .
\end{aligned}
$$

Furthermore, we obtain after taking suitable estimates

$$
p_{n+1} \leq K_{2} q_{n}^{2}+K_{3} p_{n}^{2}
$$

where $K_{2}=e^{-\widehat{A^{D} \widehat{M}} t} \int_{0}^{t} e^{\widehat{A}^{D} \widehat{M} s} \widehat{A}^{D}(-\lambda A+M(s))^{-1} N_{6} d s+$ $\left(I-\widehat{A}^{D} \widehat{A}\right) \widehat{M}^{D}(-\lambda A+M(t))^{-1} N_{6}$ and $K_{3}=e^{-\widehat{A}^{D} \widehat{M} t}$ $\int_{0}^{t} e^{\widehat{A}^{D} \widehat{M} s} \widehat{A}^{D}(-\lambda A+M(s))^{-1} N_{7} d s+\left(I-\widehat{A}^{D} \widehat{A}\right) \widehat{M}^{D}(-\lambda A+$ $M(t))^{-1} N_{7}$.

Hence we proved that the convergence rate is quadratic.
Next, we consider singular differential systems BVPs and prove the following main result.

Theorem 4. Let assumptions $\left(H_{2.1}\right)-\left(H_{2.4}\right)$ hold. Suppose further the following.

$\left(H_{3.1}\right)$ There exist $V_{0}(t), W_{0}(t) \in C^{2}\left[I, R^{n}\right]$ with $V_{0}(t) \leq W_{0}(t)$ and $\dot{V}_{0}(t) \geq \dot{W}_{0}(t)$ on $I$ and

$$
\begin{gathered}
A \ddot{V}_{0} \leq f\left(t, V_{0}, \dot{V}_{0}\right), \quad \dot{V}_{0}(0) \geq X_{0}, \quad V_{0}(b) \leq X_{1}, \\
A \ddot{W}_{0} \geq f\left(t, W_{0}, \dot{W}_{0}\right), \quad \dot{W}_{0}(0) \leq X_{0}, \quad W_{0}(b) \geq X_{1} . \\
\left(H_{3.2}\right) \text { For } X_{0} \in R^{n},\left(I-\widehat{A}^{D} \widehat{A}^{D}\right)\left(-X_{0}-\widehat{W}(0)\right)=0, \text { where } \\
\widehat{W}(t)=\left(I-\widehat{A}^{D} \widehat{A}\right) \widehat{M}^{D} \widehat{g}(t) .
\end{gathered}
$$

Then there exist monotone sequences $\left\{V_{n}\right\}$ and $\left\{W_{n}\right\}$ which converge uniformly on I to the unique solution of (1) and the convergence rate is quadratic.

Proof. Using the transformation $U=-\dot{X}$, we have that

$$
S U(t)=X(t)=X_{1}+\int_{t}^{b} U(s) d s, \quad t \in I .
$$

BVPs (1) can be transformed into IVPs of singular system:

$$
-A \dot{U}=f(t, S U,-U), \quad U(0)=-X_{0} .
$$

Let $\alpha_{0}(0)=-\dot{V}_{0}(0)$ and $\beta_{0}(0)=-\dot{W}_{0}(0)$. By using assumption $\left(H_{3.1}\right)$, we get that

$$
\alpha_{0}(t) \leq-\dot{V}_{0}(t) \leq-\dot{W}_{0}(t)=\beta_{0}(t)
$$

and $\alpha_{0}(0)=-\dot{V}_{0}(0) \leq-X_{0} \leq-\dot{W}_{0}(0)=\beta_{0}(0)$.

Noticing that $\alpha_{0}(0)=-\dot{V}_{0}(0)$, we have that

$$
\begin{array}{ll}
V_{0}(t)=V_{0}(b)+\int_{t}^{b} \alpha_{0}(s) d s, & t \in I, \\
{\left[S \alpha_{0}\right](t)=X_{1}+\int_{t}^{b} \alpha_{0}(s) d s,} & t \in I .
\end{array}
$$

Then $V_{0}(t) \leq\left[S \alpha_{0}\right](t)$ from $V_{0}(b) \leq X_{1}$, and

$$
\begin{aligned}
-A \dot{\alpha}_{0} & =A \ddot{V}_{0} \leq f\left(t, V_{0}, \dot{V}_{0}\right) \leq f\left(t, S \alpha_{0}, \dot{V}_{0}\right) \\
& =f\left(t, S \alpha_{0},-\alpha_{0}\right) .
\end{aligned}
$$

A similar argument shows that

$$
\begin{aligned}
-A \dot{\beta}_{0} & =A \ddot{W}_{0} \geq f\left(t, W_{0}, \dot{W}_{0}\right) \geq f\left(t, S \beta_{0}, \dot{W}_{0}\right) \\
& =f\left(t, S \beta_{0},-\beta_{0}\right) .
\end{aligned}
$$

By Theorem 3, there exist monotone sequences $\left\{\alpha_{n}\right\},\left\{\beta_{n}\right\}$ such that

$$
\alpha_{n} \longrightarrow U \longleftarrow \beta_{n}
$$


and the convergence rate is quadratic. Again set $V_{n}=S \alpha_{n}$ and $W_{n}=S \beta_{n}$. Then

$$
\begin{gathered}
V_{0} \leq V_{1} \leq V_{2} \leq \cdots \leq V_{n} \leq W_{n} \leq \cdots \leq W_{2} \leq W_{1} \leq W_{0} \\
V_{n} \longrightarrow U \longleftarrow W_{n}
\end{gathered}
$$

and also the convergence rate is quadratic. Noticing that

$$
\begin{aligned}
x(t)-V_{n+1}(t) & =X_{1}+\int_{t}^{b} U(s) d s-\left(X_{1}+\int_{0}^{b} \alpha_{n+1}(s) d s\right) \\
& =\int_{0}^{b}\left(U(s)-\alpha_{n+1}(s)\right) d s \\
& \leq \int_{0}^{b}\left[K_{1}\left(U(s)-\alpha_{n}(s)\right)^{2}\right] d s \\
& =K_{1}\left(x(t)-V_{n}(t)\right)^{2},
\end{aligned}
$$

we can obtain the similar result that $W_{n}$ converges quadratically to the solution of (1). The proof is complete.

\section{Conflict of Interests}

The authors declare that there is no conflict of interests regarding the publication of this paper.

\section{Authors' Contribution}

All authors completed the paper together. All authors read and approved the final paper.

\section{Acknowledgments}

The authors would like to thank the reviewers for their valuable suggestions and comments. This paper is supported by the National Natural Science Foundation of China (11271106) and the Natural Science Foundation of Hebei Province of China (A2013201232).

\section{References}

[1] V. Lakshmikantham and A. S. Vatsala, Generalized Quasilinearization for Nonlinear Problems, Kluwer Academic Publishers, Dordrecht, The Netherlands, 1998.

[2] A. R. Abd-Ellateef Kamar, G. M. Attia, K. Vajravelu, and M. Mosaad, "Generalized quasilinearization for singular system of differential equations," Applied Mathematics and Computation, vol. 114, no. 1, pp. 69-74, 2000.

[3] W. Wang and Y. Q. Liu, "Monotone iterative for boundary value problem of second order singular dierential system," Journal of Systems Science and Systems Engineering, vol. 4, no. 4, pp. 266272, 1995.

[4] S. L. Campbell, Singular Systems of Differential Equations, Pitman Advanced Publishing Program, London, UK, 1980.

[5] A. S. Vatsala, "Monotone iterative technique for singular systems of differential equations," in Nonlinear Analysis and
Applications, vol. 109 of Lecture Notes in Pure and Applied Mathematics, pp. 579-582, Dekker, New York, NY, USA, 1987. 


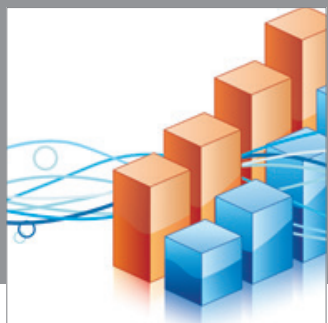

Advances in

Operations Research

mansans

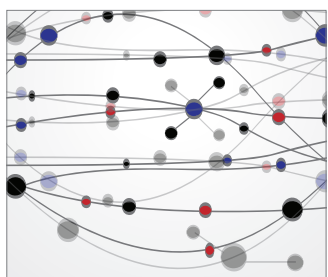

The Scientific World Journal
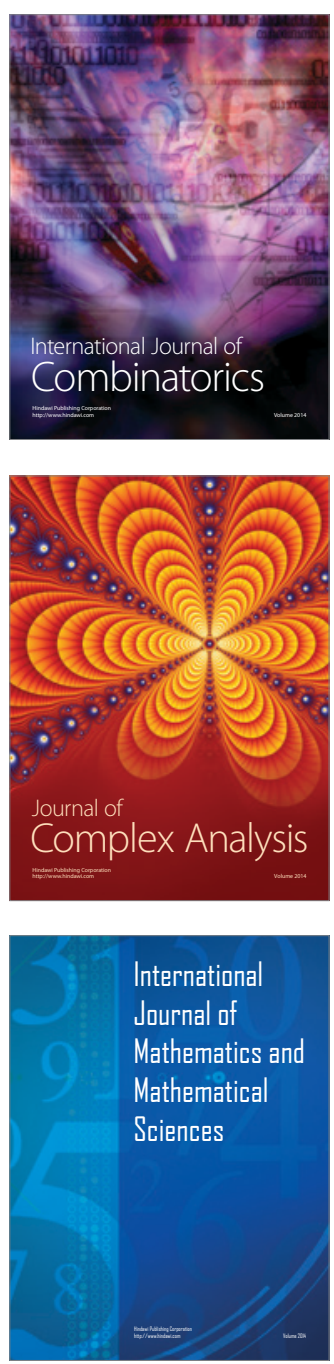
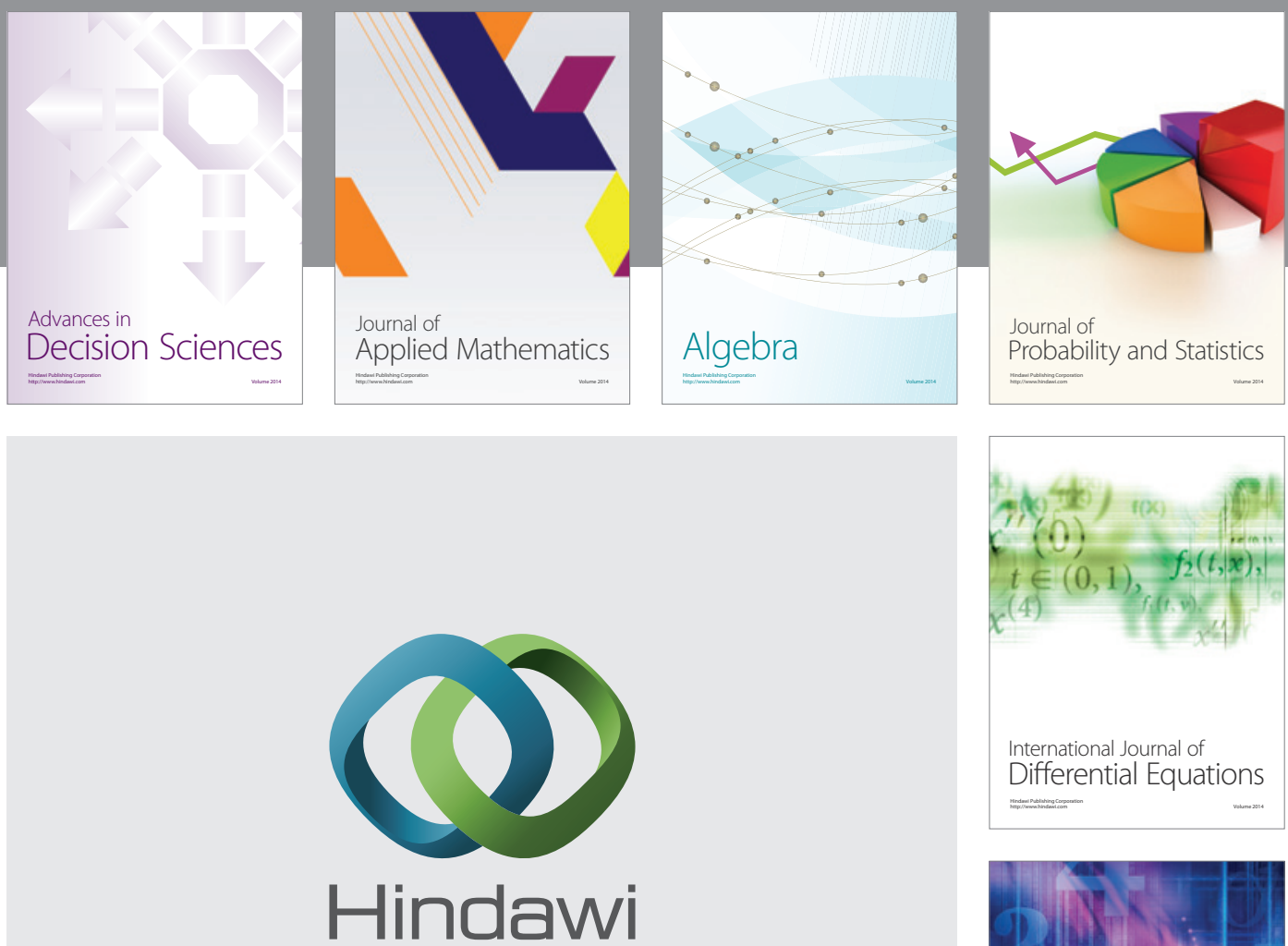

Submit your manuscripts at http://www.hindawi.com
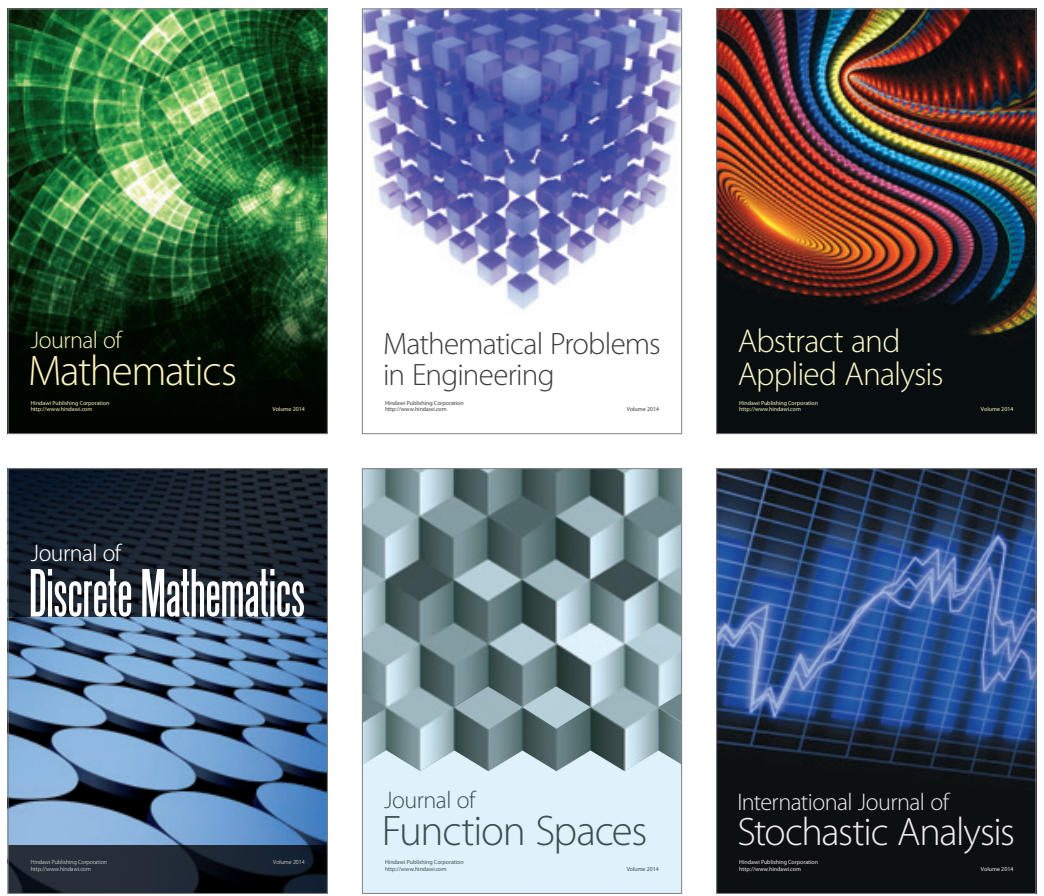

Journal of

Function Spaces

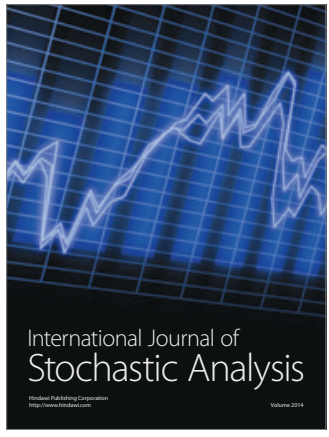

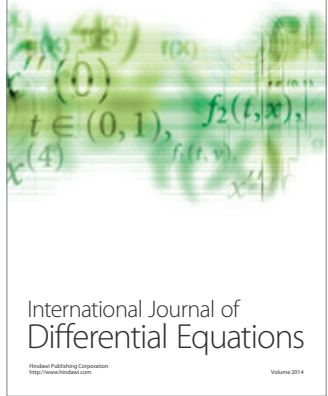
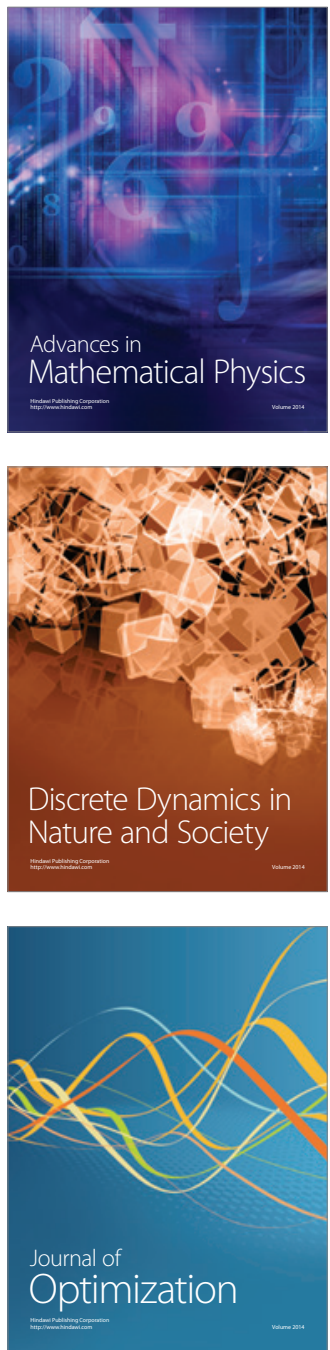